\title{
Effects of human umbilical cord-derived mesenchymal stem cells on hematologic malignancies
}

\author{
QIAN LII ${ }^{1-3}$, YILIN PANG ${ }^{1,2,4}$, TINGTING LIU ${ }^{1,2}$, YONGYONG TANG ${ }^{1,2}$, \\ JING XIE ${ }^{1,2}$, BIN ZHANG ${ }^{1,2}$ and HU CHEN $^{1,2}$ \\ ${ }^{1}$ Department of Hematopoietic Stem Cell Transplantation; ${ }^{2}$ Cell and Gene Therapy Center, Affiliated Hospital of \\ Academy of Military Medical Sciences, Beijing 100071; ${ }^{3}$ Department of Oncology, The Army General \\ Hospital, Beijing 100010; ${ }^{4}$ Department of Emergency, Beijing Children's Hospital, \\ Capital Medical University, Beijing 100071, P.R. China
}

Received March 3, 2016; Accepted July 5, 2017

DOI: $10.3892 / \mathrm{ol} .2018 .8254$

\begin{abstract}
Mesenchymal stem cells (MSCs) have been used in hematopoietic stem cell transplantation for years. However, the safety of MSCs applied in various types of hematologic malignancy has not been comprehensively explored. In the present study, the effects of human umbilical cord-derived mesenchymal stem cells (hUC-MSCs) on six representative hematologic malignancy cell lines were explored, including leukemia, multiple myeloma and lymphoma cells. Direct and indirect co-culture models were established, and cell proliferation was assessed by carboxyfluorescein diacetate succinimidyl ester staining. A cytometric bead array cytokine kit was used to quantify cytokines. The expression of interleukin (IL)-6 receptor elements on tumor cells was detected by reverse transcription-polymerase chain reaction and flow cytometry, and the effects of exogenous IL- 6 on cell proliferation were determined using a Cell Counting kit- 8 assay. The results demonstrated that hUC-MSCs inhibited the proliferation of most of the cell lines examined (THP-1, HL-60, K562 and RPMI-8226), but promoted the proliferation of Raji cells. In addition, hUC-MSCs secreted abundant IL-6, promoted the secretion of IL-10 by RPMI-8226 and Raji cells, and inhibited the secretion of tumor necrosis factor- $\alpha$ by THP- 1 cells. These data indicate a varied effect of hUC-MSCs on various types of hematologic malignancy, including distinct mechanisms of cell-to-cell contact and cytokines. Researchers applying hUC-MSCs in lymphoma should be aware of a potential tumor growth-promoting effect.
\end{abstract}

Correspondence to: $\mathrm{Dr} \mathrm{Hu}$ Chen or Professor Bin Zhang, Department of Hematopoietic Stem Cell Transplantation, Affiliated Hospital of Academy of Military Medical Sciences, 8 Dongda Street, Fengtai, Beijing 100071, P.R. China

E-mail: chenhu217@aliyun.com

E-mail: zb307ctc@163.com

Key words: mesenchymal stem cells, hematologic malignancy, proliferation, cytokines, interleukin-6

\section{Introduction}

Mesenchymal stem cells (MSCs) are a type of adult stem cell that exhibits self-renewal capacity and differentiation potential; they occur in the bone marrow (BM), muscle, adipose tissue, bone, placenta and umbilical cord (1). Compared with other sources, MSCs from the umbilical cord (hUC-MSCs) may be the most optimal source on account of their abundant supply, improved expandability, lower probability of microbial contamination and reduced ethical limitations relative to MSCs from other sources (2).

MSCs have potential applicability in tissue damage, autoimmune diseases, neurodegenerative diseases, diabetes and cancer therapy (3-6). Furthermore, as they may support hematopoiesis and immune regulation, MSCs have been applied in hematopoietic stem cell (HSC) transplantation for years (7). A stem cell treatment used in the treatment of severe graft-vs.-host disease (GVHD) in children, Prochymal, was approved in Canada and New Zealand in 2012 (8). However, there is controversy regarding the application of MSCs in patients with a malignancy due to their potential cancer-promoting effects. Different groups have arrived at opposite conclusions concerning the effect of MSCs on tumors in vivo and in vitro (9-11). Our previous study indicated that the co-transplantation of HSCs and MSCs may prevent GVHD, but may simultaneously increase the relapse rate in patients with hematologic malignancy relative to the transplantation of HSCs alone (12). Accumulating evidence suggests that the interaction between MSCs and tumors is regulated by multiple factors, particularly in vivo: MSCs may migrate to the tumor sites and promote or inhibit the growth, invasion and metastasis of tumors $(9,10)$ through cell-to-cell contact (13) or paracrine secretion (14). They not only regulate the cell cycle and apoptosis of tumor cells directly (15), but also induce immune tolerance by secreting immune regulatory factors, including indoleamine-2,3-dioxygenase, nitrogen monoxide, and interleukin (IL)-6, which may facilitate the immune escape of a tumor $(16,17)$.

Prior studies on the effects of MSCs on tumors have predominantly focused on solid tumors; the effect of MSCs on hematologic malignancies has yet to be characterized. In the 
present study, the effects of hUC-MSCs on the proliferation of six cell lines representative of various types of hematologic malignancy, including leukemia (THP-1, HL-60, K562 and Jurkat), multiple myeloma (RPMI-8226) and lymphoma (Raji), were explored, including through direct and indirect co-culture models; the potential mechanisms of cell-to-cell contact and cytokines were then discussed.

\section{Materials and methods}

Isolation and culture of hUC-MSCs. The present study was approved by the Institutional Review Board of the Affiliated Hospital of the Academy of Military Medical Sciences (Beijing, China; protocol \#2010-05-60). Written informed consent forms were obtained from the healthy umbilical cord donors. The isolation and identification of hUC-MSCs were performed as described previously (18); flow cytometry was used to identify hUC-MSCs, which were positive for cluster of differentiation (CD) 73, 90, 105 and 166, and negative for hematopoietic markers, including CD45, 14 and 34, and major histocompatibility complex class II-DR. Resuspended cells were plated at a density of $5 \times 10^{5} / \mathrm{ml}$ and maintained in DMEM/F12 (Hyclone; GE Healthcare, Chicago, IL, USA) supplemented with $10 \%$ fetal bovine serum (FBS; Gibco; Thermo Fisher Scientific, Inc., Waltham, MA, USA) in a humidified atmosphere with $5 \% \mathrm{CO}_{2}$ at $37^{\circ} \mathrm{C}$. The ability of hUC-MSCs to differentiate into osteoblasts and adipocytes was confirmed prior to use, as previously described (18). Cells were used in the present study subsequent to 5-6 passages.

Culture and carboxyfluorescein diacetate succinimidyl ester (CFSE) staining of tumor cell lines. THP-1 and HL-60 acute myeloid leukemia (AML) cells, K562 chronic myeloid leukemia (CML) cells, Jurkat acute lymphoblastic leukemia (ALL) cells, RPMI-8226 multiple myeloma (MM) cells and Raji Burkitt's lymphoma cells were obtained from the China Infrastructure of Cell Line Resources (Beijing, China). All cell types were initially maintained in RPMI-1640 medium (Hyclone; GE Healthcare) supplemented with 10\% FBS at $37^{\circ} \mathrm{C}$ in a humidified atmosphere of $5 \% \mathrm{CO}_{2}$. The medium was changed every three days. To be stained with CFSE (Sigma-Aldrich; Merck KGaA, Darmstadt, Germany), tumor cells were washed three times and resuspended in RPMI-1640 medium, then mixed with an equal volume of $5 \mu \mathrm{mol} / \mathrm{l} \mathrm{CFSE}$ and incubated for $10 \mathrm{~min}$ at $37^{\circ} \mathrm{C}$. The reaction was terminated by adding an equal volume of FBS for $10 \mathrm{~min}$ at $4^{\circ} \mathrm{C}$. Finally, cells were washed and resuspended in DMEM/F12 medium supplemented with $10 \%$ FBS for experiments.

Direct and indirect co-culture models and assessment of cell proliferation. hUC-MSCs and CFSE-stained tumor cells were co-cultured in 6-well plates at the ratios of 2:1, 1:1, 1:4 and $1: 16$, or in the lower and upper compartments, respectively, of transwell plates at 2:1, 1:1 and 1:4. Tumor cells alone served as controls. Cells were cultured in DMEM/F12 medium supplemented with $10 \% \mathrm{FBS}$ for $72 \mathrm{~h}$ at $37^{\circ} \mathrm{C}$, and then tumor cells were harvested; $10^{5} \mathrm{CFSE}$-positive cells from each sample were acquired and the FL1 fluorescence intensity was detected with an Accuri C6 flow cytometer (BD Biosciences, Franklin Lakes, NJ, USA). Data were analyzed using FCS Express
4.0 software (De Novo Software, Glendale, CA, USA). The experiment was repeated three times.

Measurement of cytokines by cytometric bead array (CBA). hUC-MSCs alone, tumor cells alone or both at an equal concentration were cultured in 6 -well plates for $72 \mathrm{~h}$ at $37^{\circ} \mathrm{C}$. The supernatants were harvested and centrifuged $(2,210 \mathrm{x} \mathrm{g}$ for $5 \mathrm{~min}$ at room temperature) to remove precipitation. Cell-free supernatants were collected and frozen at $-80^{\circ} \mathrm{C}$. The IL-2, $-4,-6$ and -10 , tumor necrosis factor (TNF)- $\alpha$ and interferon (IFN) $-\gamma$ content was measured according to the protocol for the CBA cytokine kit (BD Biosciences). Briefly, cytokine standards were prepared and cytokine capture beads were mixed; $50 \mu 1$ of mixed capture beads, phycoerythrin detection reagent and samples or standards were then added to all assay tubes. The assay tubes were incubated for $3 \mathrm{~h}$ at room temperature in the dark, then washed and centrifuged. The supernatant was carefully aspirated and discarded, and bead pellets were resuspended with $300 \mu \mathrm{l}$ wash buffer. Data were obtained and analyzed using CBA analysis with CellQuest software (version 3.3) and CBC software (version 3.0) (BD Biosciences). The experiment was repeated three times.

Detection of the expression of IL-6, IL-6R $\alpha$ and IL-6 signal transducer (gp130) mRNAs by reverse transcription-polymerase chain reaction $(R T-P C R)$. Total RNA was extracted using the Pure RNA rapid extraction kit (Biomed, Beijing, China) according to the manufacturer's protocol and reverse transcribed using the First-Strand Synthesis system (Thermo Fisher Scientific, Inc.). Reverse transcription was performed at $42^{\circ} \mathrm{C}$ for $1 \mathrm{~h}$ then $25^{\circ} \mathrm{C}$ for $5 \mathrm{~min}$. PCR was performed using GAPDH as internal control; primer sequences and thermocycling conditions are listed in Table I. The primers and Taq PCR Master MIX were supplied by Biomed (Beijing, China). All products were evaluated by electrophoresis on $1 \%$ agarose gel. The experiment was repeated three times.

Detection of the expression of IL-6R $\alpha$ and gp130 proteins by flow cytometry. Flow cytometry was used to identify the expression of IL-6R $\alpha$ and gp130 proteins on the membranes of tumor cells, which were labeled with monoclonal antibodies against IL-6R $\alpha$ conjugated to allophycocyanin (APC; APC Mouse Anti-Human CD126, catalog no. 562090, BD Biosciences) and gp130 conjugated to phycoerythrin (PE; PE Mouse Anti-Human CD130, catalog no. 555757, BD Biosciences). Tumor cells were incubated with the 1:100 diluted antibodies for $15 \mathrm{~min}$ at room temperature in the dark. At least 10,000 events were acquired on the BD Accuri C6 and data were analyzed using the Accuri C6 software (version 1.0, BD Biosciences). The experiment was repeated three times.

Assessment of effects of IL-6 on cell proliferation. Tumor cells were seeded in 96 -well plates at $1 \times 10^{4}$ cells/well with increasing concentrations of recombinant human IL-6 (0, 10, 20, 50 and $100 \mathrm{ng} / \mathrm{ml}$; R\&D Systems, Inc., Minneapolis, $\mathrm{MN}, \mathrm{USA}$ ) and incubated at $37^{\circ} \mathrm{C}$. At $72 \mathrm{~h}, 10 \mu \mathrm{l}$ Cell Counting kit-8 (CCK-8) reagent (Dojindo Molecular Technologies, Inc., Kumamoto, Japan) was added to the wells for $2-5 \mathrm{~h}$ at $37^{\circ} \mathrm{C}$, and the absorbance at $450 \mathrm{~nm}$ was measured with an iMark 
Table I. Primers for PCR.

\begin{tabular}{lllc}
\hline Gene & \multicolumn{1}{c}{ Primer sequences (5'-3') } & \multicolumn{1}{c}{ Reaction condition } & Size (bp) \\
\hline IL-6 receptor $\alpha$ chain & F: CATTGCCATTGTTCTGAGGTTC & $94^{\circ} \mathrm{C}$ for $30 \mathrm{sec}, 60^{\circ} \mathrm{C}$ for $30 \mathrm{sec}$, & 280 \\
& R: GTGCCACCCAGCCAGCTATC & $72^{\circ} \mathrm{C}$ for $60 \mathrm{sec}, 40 \mathrm{cycles}$ & \\
IL-6 signal transducer & F: CATAGTCGTGCCTGTTTGCTTAG & $94^{\circ} \mathrm{C}$ for $30 \mathrm{sec}, 62^{\circ} \mathrm{C}$ for $30 \mathrm{sec}$, & 527 \\
& R: GATCTTCTGGCCGCTCCTC & $72^{\circ} \mathrm{C}$ for $60 \mathrm{sec}, 40 \mathrm{cycles}$ & \\
IL-6 & F: CCCCAGTACCCCCAGGAGAAGA & $94^{\circ} \mathrm{C}$ for $30 \mathrm{sec}, 57^{\circ} \mathrm{C}$ for $30 \mathrm{sec}$, & 349 \\
& R: GCTGCGCAGAATGAGATGAGTTGT & $72^{\circ} \mathrm{C}$ for $60 \mathrm{sec}, 40 \mathrm{cycles}$ & \\
GAPDH & F: GAGTCAACGGATTTGGTCGT & $94^{\circ} \mathrm{C}$ for $30 \mathrm{sec}, 57^{\circ} \mathrm{C}$ for $30 \mathrm{sec}$, & 238
\end{tabular}

IL-6, interleukin-6; F, forward; R, reverse.

microplate reader (Bio-Rad Laboratories, Inc., Hercules, CA, USA). The experiment was repeated three times.

Statistics. The experimental data are presented as mean values, with bars representing the standard deviation. Data were analyzed using GraphPad Prism version 6.0 (GraphPad Software, Inc., La Jolla, CA, USA) and SPSS version 19 (IBM Corp., Armonk, NY, USA) software. Determinations of statistical significance were performed using Student's t-test for comparisons of two groups or an analysis of variance (ANOVA) followed by Tukey's post-hoc test for comparisons of multiple groups. $\mathrm{P}<0.05$ was considered to indicate a statistically significant difference.

\section{Results}

hUC-MSCs affect cells from different types of hematologic malignancy differently in co-culture. hUC-MSCs and tumor cells were co-cultured in 6-well plates for $72 \mathrm{~h}$. The mean fluorescence intensity (MFI) of cells stained with CFSE is gradually attenuated in the process of proliferation. Increases or decreases in MFI relative to a control indicated the inhibition or promotion of tumor cell proliferation by hUC-MSCs, respectively.

For the AML cell lines THP-1 (Fig. 1A) and HL-60 (Fig. 1B), and the CML cell line K562 (Fig. 1C), MFI in the experimental groups was higher than in the control groups, and increased with the concentration of hUC-MSCs, indicating that the hUC-MSCs inhibited proliferation in a dose-dependent manner. hUC-MSCs only promoted proliferation when added at a 1:1 concentration to the ALL Jurkat cells (Fig. 1D). In the MM cell line RPMI-8226 (Fig. 1E), hUC-MSCs inhibited proliferation at all doses. hUC-MSCs significantly promoted the proliferation of the Burkitt's lymphoma cell line Raji (Fig. 1F). These data indicated that hUC-MSCs have various effects on cancer cell proliferation. In summary, hUC-MSCs inhibited the proliferation of THP-1, HL-60, K562 and RPMI-8226 cells, but promoted Raji cell proliferation (Table II).

hUC-MSCs affect cells from different types of hematologic malignancy differently in transwells. To explore the roles of cell-to-cell contact and cytokines secreted by hUC-MSCs, CFSE-stained tumor cells were separated from hUC-MSCs by transwells and cultured for $72 \mathrm{~h}$. Cell proliferation was assessed as previously described.

For the leukemia cell lines THP-1 (Fig. 2A), HL-60 (Fig. 2B) and K562 (Fig. 2C), hUC-MSCs inhibited proliferation, consistent with the previous results, suggesting that inhibitory cytokines were involved in the effect. However, for the ALL cell line Jurkat (Fig. 2D), hUC-MSCs inhibited cell proliferation in transwells, whereas there had been almost no effect on proliferation observed when co-cultured directly; we speculated that the growth promotion caused by cell-to-cell contact may have offset the inhibitory effect of cytokines. For the MM cell line RPMI-8226, consistent with previous results, hUC-MSCs inhibited proliferation (Fig. 2E) in a dose-dependent manner, potentially due to inhibitory cytokines. For the Burkitt's lymphoma cell line Raji (Fig. 2F), although hUC-MSCs significantly promoted cell proliferation in direct co-culture, they had no effect in transwells, implying that cell-to-cell contact may have been a dominant mechanism for growth promotion in Raji cells.

The results revealed that hUC-MSCs have distinct effects on different types of hematologic malignancies, likely due to diverse mechanisms. In summary, growth inhibition caused by hUC-MSCs in THP-1, HL-60, K562 and RPMI-8226 cells is likely to be associated with inhibitory cytokines, whereas the growth promotion of Raji is likely to be caused by cell-to-cell contact. For Jurkat cells, the effect varied depending on the concentration of hUC-MSCs (Table II).

HUC-MSCs secrete abundant IL-6 and affect the secretion of tumor cells. In order to investigate the specific cytokines involved in the effects described in the previous results, the levels of IL-2, -4, -6 and -10 , and TNF- $\alpha$ and IFN- $\gamma$ secreted by hUC-MSCs were determined using CBA; it was observed that the hUC-MSCs secreted abundant IL-6, whereas the secretion of IL-2, IL-4, IL-10, TNF- $\alpha$ and IFN- $\gamma$ was limited (Fig. 3A).

These cytokines were then detected in the supernatants of the tumor cells cultured alone, and co-cultured 1:1 with hUC-MSCs, for $72 \mathrm{~h}$. It was confirmed that hUC-MSCs secreted a high level of IL-6 in the co-culture supernatants. In addition, it was observed that hUC-MSCs affected the secretion by tumor cells. As presented in Fig. 3B, THP-1 cells secreted a small amount of IL- 6 and TNF- $\alpha$, and the secretion of TNF- $\alpha$ was inhibited when co-cultured with hUC-MSCs; 

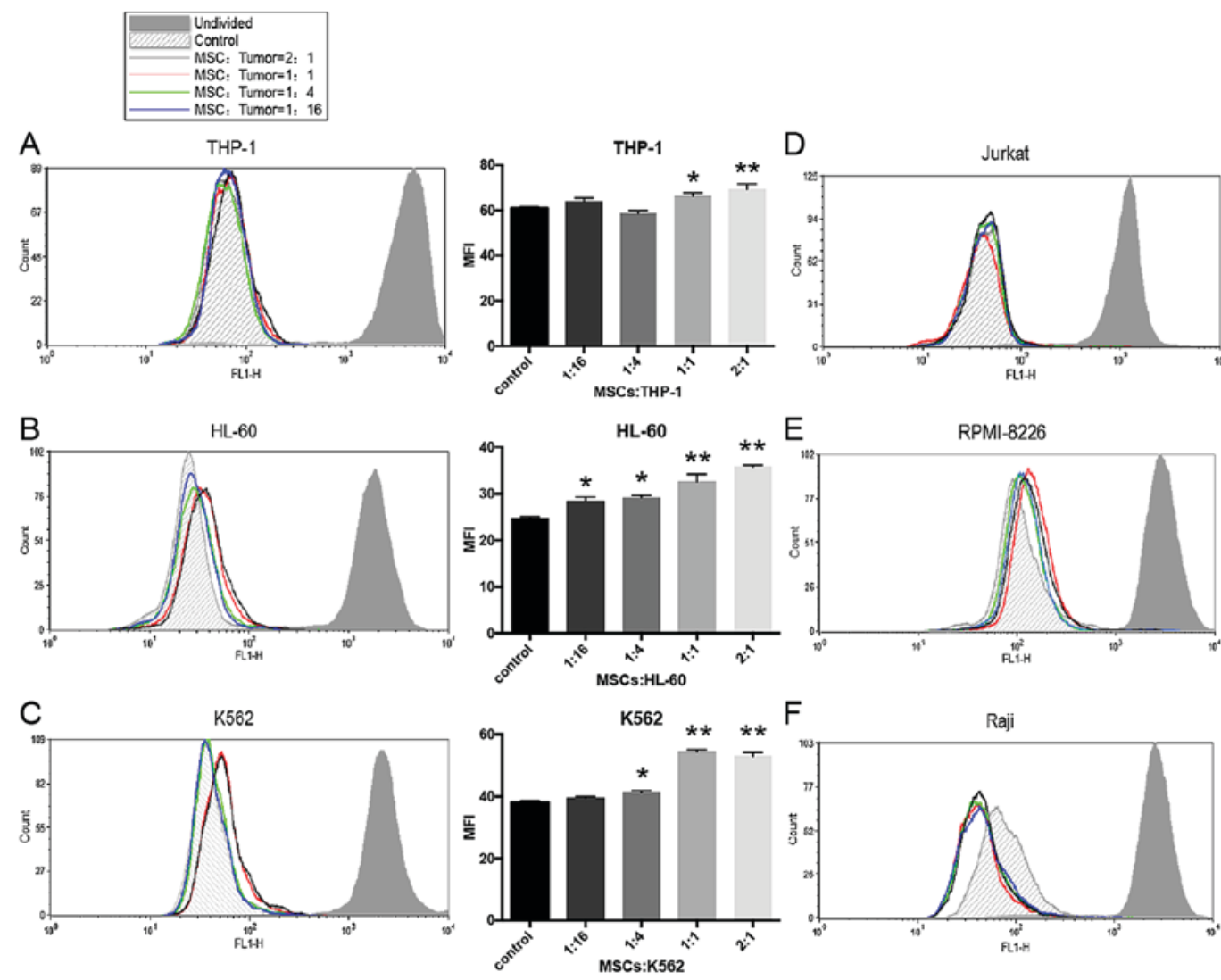
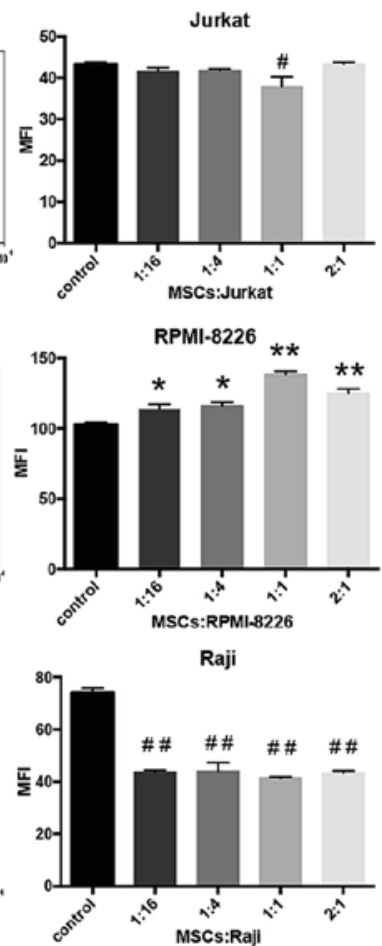

Figure 1. hUC-MSCs affect the proliferation of tumor cells in direct co-culture. hUC-MSCs and carboxyfluorescein diacetate succinimidyl ester-stained tumor cells, including (A) THP-1, (B) HL-60, (C) K562, (D) Jurkat, (E) RPMI-8226 and (F) Raji cells, were co-cultured in 6-well plates for 72 h; the MFI of tumor cells was assayed by flow cytometry. Left: Flow cytometry profiles of one representative experiment. Right: MFI of tumor cells from 3 independent experiments. Data are expressed as the mean \pm standard deviation. ${ }^{*} \mathrm{P}<0.05,{ }^{* *} \mathrm{P}<0.01$ inhibition of tumor cell growth compared with the control group; ${ }^{\#} \mathrm{P}<0.05$, ${ }^{\# \#} \mathrm{P}<0.01$ promotion of tumor cell growth compared with the control group. hUC-MSCs, human umbilical cord-derived mesenchymal stem cells; MFI, mean fluorescence intensity.

K562 cells secreted a small amount of IL-6; RPMI-8226 and Raji cells secreted IL-10, which was promoted by hUC-MSCs. It was inferred that hUC-MSCs may promote the secretion of IL-10 and inhibit the secretion of TNF- $\alpha$ by tumor cells.

The expression of IL-6R mRNA and protein varies between cells from different types of hematologic malignancy. IL-6 must bind with the receptor composed of IL-6R $\alpha$ and gp130 to activate the downstream signaling pathway, which may affect the development of multiple types of tumor (19). In order to determine whether tumor cell lines were affected by IL-6, the expression of IL-6, IL-6R $\alpha$ and gp130 mRNA in tumor cell lines was assessed using RT-PCR; it was observed that HL-60, RPMI-8226 and THP-1 cells expressed IL-6R $\alpha$ and gp130 mRNA, K562 and Jurkat cells expressed gp130 mRNA, whereas Raji cells expressed neither (Fig. 4A; Table II). In addition, the expression of IL- 6 mRNA by THP-1 and K562 cells was identified, consistent with the conclusion from CBA that they could secrete a small amount of IL-6.

Subsequently, the expression of IL-6R $\alpha$ and gp130 protein on the membrane of the tumor cells was detected by flow cytometry. The results were consistent with the RT-PCR results: THP-1,HL-60 and RPMI-8226 cells expressed IL-6R $\alpha$ and gp130 protein, and K562 and Jurkat cells expressed gp130 protein, whereas Raji cells expressed neither (Fig. 4B; Table II).
Exogenous IL-6 promotes the proliferation of tumor cells expressing $I L-6 R$. To determine whether IL-6 is a mediator of the effects of hUC-MSCs on tumor cells, the effects of exogenous IL- 6 on the proliferation of tumor cells were examined. Cells were cultured in medium supplemented with increasing concentrations of recombinant human IL- 6 for $72 \mathrm{~h}$, and the proliferation was assessed by a CCK-8 assay. As presented in Fig. 5, exogenous IL-6 significantly promoted the proliferation of THP-1 and HL-60 cells $(\mathrm{P}<0.05)$, but exhibited no significant effect on K562, Jurkat, RPMI-8226 and Raji cells (Table II). Combined with the previous results, it can be concluded that IL- 6 is not a key mediator of the effects of hUC-MSCs; the stimulation of IL- 6 on THP-1 and HL-60 cells may be offset by other inhibitory cytokines when co-cultured with hUC-MSCs.

\section{Discussion}

In the past, MSCs were most commonly isolated from BM. However, the aspiration of BM involves invasive procedures, limiting its availability. Therefore, an alternative source of MSCs is clinically valuable. It has been demonstrated that hUC-MSCs also exhibit potential applicability for allogeneic HSC transplantation similar to that of BM-MSCs (20); hUC-MSCs have subsequently become a new focus for stem cell research due to their abundant supply. However, considering 
Table II. The expression of IL-6R and the effects of exogenous IL-6 and hUC-MSCs on tumor cells.

\begin{tabular}{llllllll}
\hline & & \multicolumn{2}{c}{ IL-6R } & & & \multicolumn{2}{c}{ hUC-MSCs } \\
\cline { 3 - 4 } Tumor type & Cell line & IL-6R $\alpha$ & gp130 & Exogenous IL-6 & Co-culture & Transwell \\
\hline Acute myeloid leukemia & THP-1 & + & + & $\mathrm{U}$ & $\mathrm{D}$ & $\mathrm{D}$ \\
Acute promyeloid leukemia & HL-60 & + & + & $\mathrm{U}$ & $\mathrm{D}$ & $\mathrm{D}$ \\
Chronic myeloid leukemia & K562 & - & + & $\mathrm{N}$ & $\mathrm{D}$ & $\mathrm{D}$ \\
Acute lymphoblastic leukemia & Jurkat & - & + & $\mathrm{N}$ & $\mathrm{N}$ & $\mathrm{D}$ \\
Multiple myeloma & RPMI-8226 & + & + & $\mathrm{N}$ & $\mathrm{D}$ & $\mathrm{D}$ \\
Burkitt's lymphoma & Raji & - & - & $\mathrm{N}$ & $\mathrm{U}$ & $\mathrm{N}$
\end{tabular}

IL-6, interleukin-6; IL-6R, interleukin-6 receptor; IL-6R $\alpha$, interleukin-6 receptor $\alpha$ chain; gp130, interleukin 6 signal transducer; hUC-MSCs, human umbilical cord-derived mesenchymal stem cells; +, cells express the corresponding protein; -, cells do not express the corresponding protein; $\mathrm{U}$, proliferation of tumor cells promoted; D, proliferation of tumor cells inhibited; N, proliferation of tumor cells unaffected.
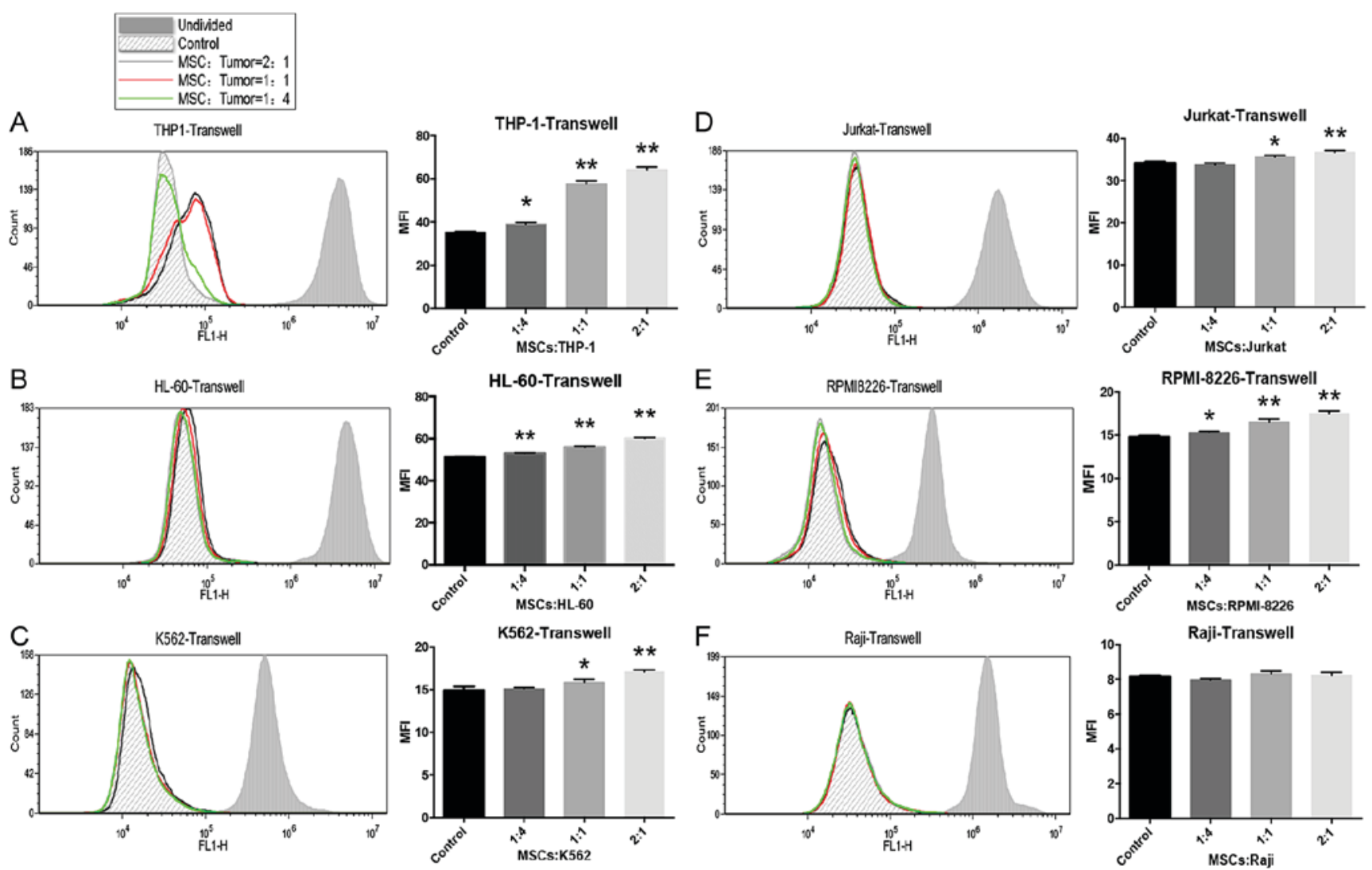

Figure 2. hUC-MSCs affect the proliferation of tumor cells in transwells. hUC-MSCs and carboxyfluorescein diacetate succinimidyl ester-stained tumor cells, including (A) THP-1, (B) HL-60, (C) K562, (D) Jurkat, (E) RPMI-8226 and (F) Raji cells, were separately cultured in the lower and upper compartments of transwells for $72 \mathrm{~h}$, and the MFI of tumor cells was assayed by flow cytometry. Left: Flow cytometry profiles of one representative experiment. Right: MFI of tumor cells from 3 independent experiments. Data are expressed as the mean \pm standard deviation. ${ }^{*} \mathrm{P}<0.05,{ }^{* * *} \mathrm{P}<0.01$ compared with the control group. hUC-MSCs, human umbilical cord-derived mesenchymal stem cells; MFI, mean fluorescence intensity.

the emerging evidence that BM-MSCs may increase the risk of cancer relapse (10-12), it remains necessary to evaluate the safety of hUC-MSCs prior to their clinical application.

At present, there is no definite conclusion about the pro- or anti-tumorigenic effect of MSCs, which may be influenced by the MSC source, tumor type and experimental conditions. In the present study, the effects of hUC-MSCs on leukemia, MM and lymphoma cells were investigated simultaneously in order to exclude the factors of different MSC sources and experimental conditions; the whole results are summarized in Table II. It was revealed that hUC-MSCs predominantly exerted an inhibitory effect on leukemia cells; previous research has also demonstrated that MSCs from other sources, including BM and umbilical cord blood, may inhibit the development of leukemia by affecting the cell cycle and apoptosis (21-23). It may be inferred that this inhibition is associated with specific 

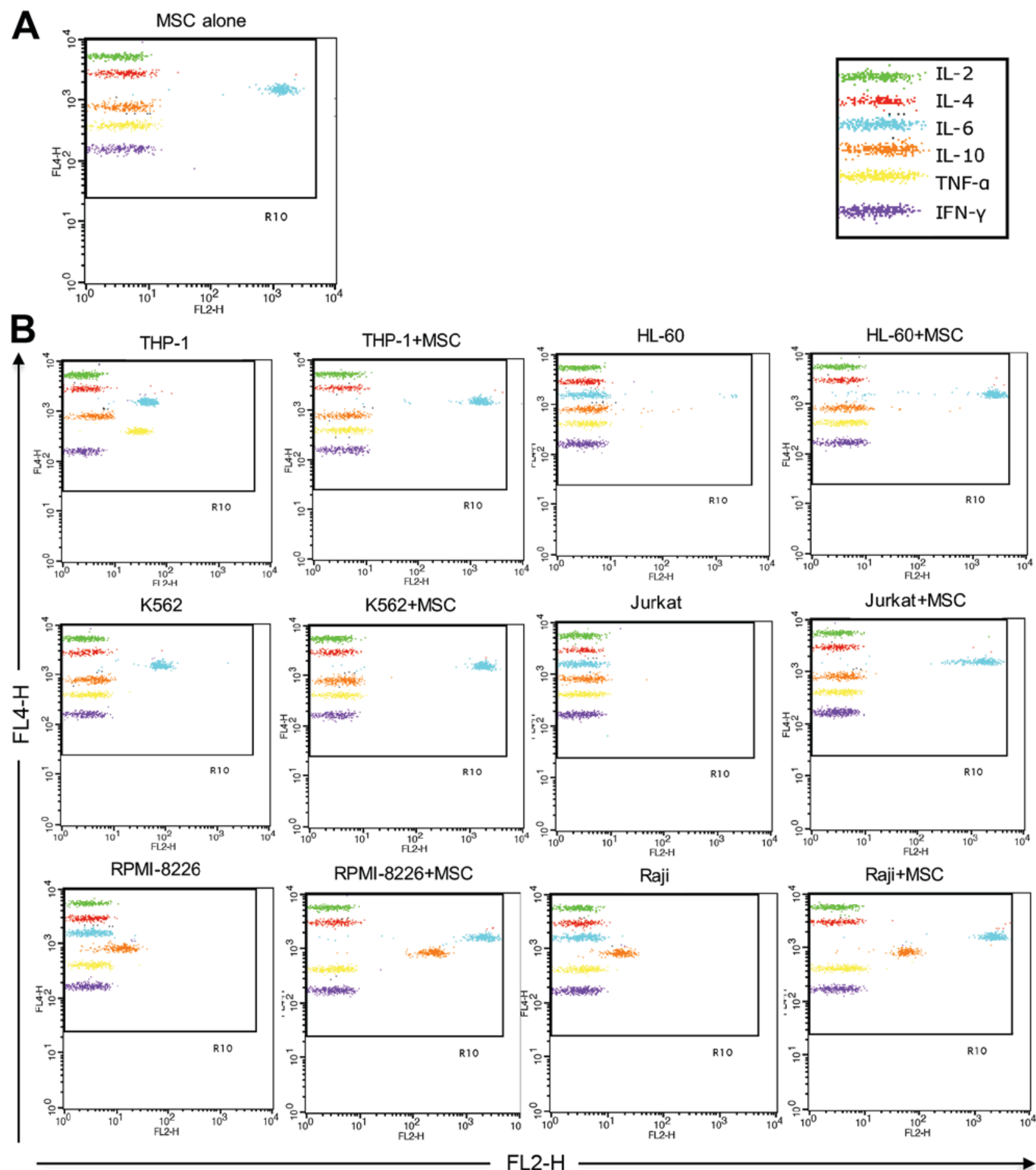

Figure 3. hUC-MSCs secrete abundant IL- 6 and affect the secretion of tumor cells. (A) The IL-2, $-4,-6$ and -10 , and TNF- $\alpha$ and IFN- $\gamma$ content of the supernatant of hUC-MSCs cultured alone were measured with a cytometric bead array. With the exception of IL-6, cytokines were almost undetectable. (B) The cytokine content in the supernatants of tumor cells cultured alone or co-cultured with hUC-MSCs at a 1:1 concentration for $72 \mathrm{~h}$ were measured. hUC-MSCs inhibited the secretion of TNF- $\alpha$ by THP- 1 cells, and promote the secretion of IL-10 by RPMI- 8226 and Raji cells. The experiments were repeated three times. hUC-MSC, human umbilical cord-derived mesenchymal stem cells; IL, interleukin; TNF- $\alpha$, tumor necrosis factor- $\alpha$; IFN- $\gamma$, interferon- $\gamma$.

inhibitory cytokines secreted by hUC-MSCs, as cytokine receptors are expressed on the majority of leukemia cells. However, a specific cytokine was not confirmed in the present study by the subsequent experiments, although the effect was likely to have been independent of IL-6, as it promoted or did not affect cell proliferation in the present study. Zhu et al (24) previously demonstrated that MSCs inhibited the proliferation of K562 by secreting Dickkopf Wnt signaling pathway inhibitor 1 (DKK-1) to negatively regulate the Wnt signaling pathway. Whether DKK-1 is also associated with other types of leukemia requires further investigation.

IL-6 serves an important function in MM, as it may promote the differentiation of $\mathrm{B}$ cells into plasma cells and accelerate MM development by stimulating proliferation and inhibiting the apoptosis of malignant plasma cells $(25,26)$. The application of IL-6 monoclonal antibody to treat MM 

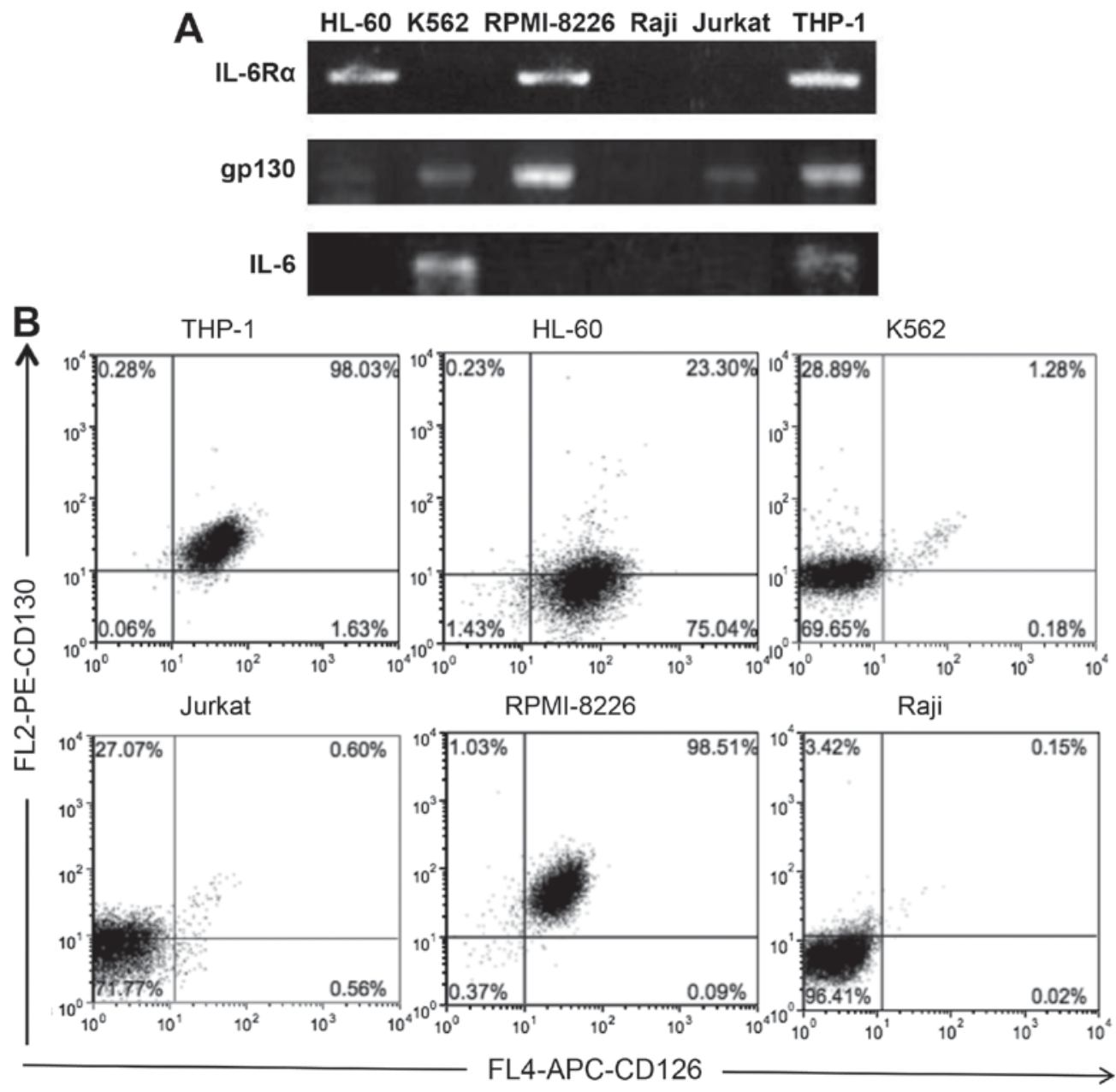

Figure 4. The expression of IL-6R $\alpha$ and gp130 mRNA and protein in the tumor cells. (A) The expression of IL-6, IL-6R $\alpha$ and gp130 mRNA in the tumor cells was detected by reverse transcription-polymerase chain reaction. THP-1 expressed all three, HL-60 and RPMI-8226 expressed IL-6R $\alpha$ and gp130, K562 expressed gp130 and IL-6, Jurkat expressed gp130 only, and Raji expressed none. (B) The expression of IL-6R $\alpha$ and gp130 proteins on tumor cells was detected by flow cytometry. The x-axis represents IL-6R $\alpha$ and the y-axis represents gp130. THP-1, HL-60 and RPMI-8226 expressed both; K562 and Jurkat expressed gp130 only; Raji expressed neither. The experiments were repeated three times. IL-6R $\alpha$, interleukin- 6 receptor $\alpha$; gp130, interleukin 6 signal transducer; IL-6, interleukin 6.
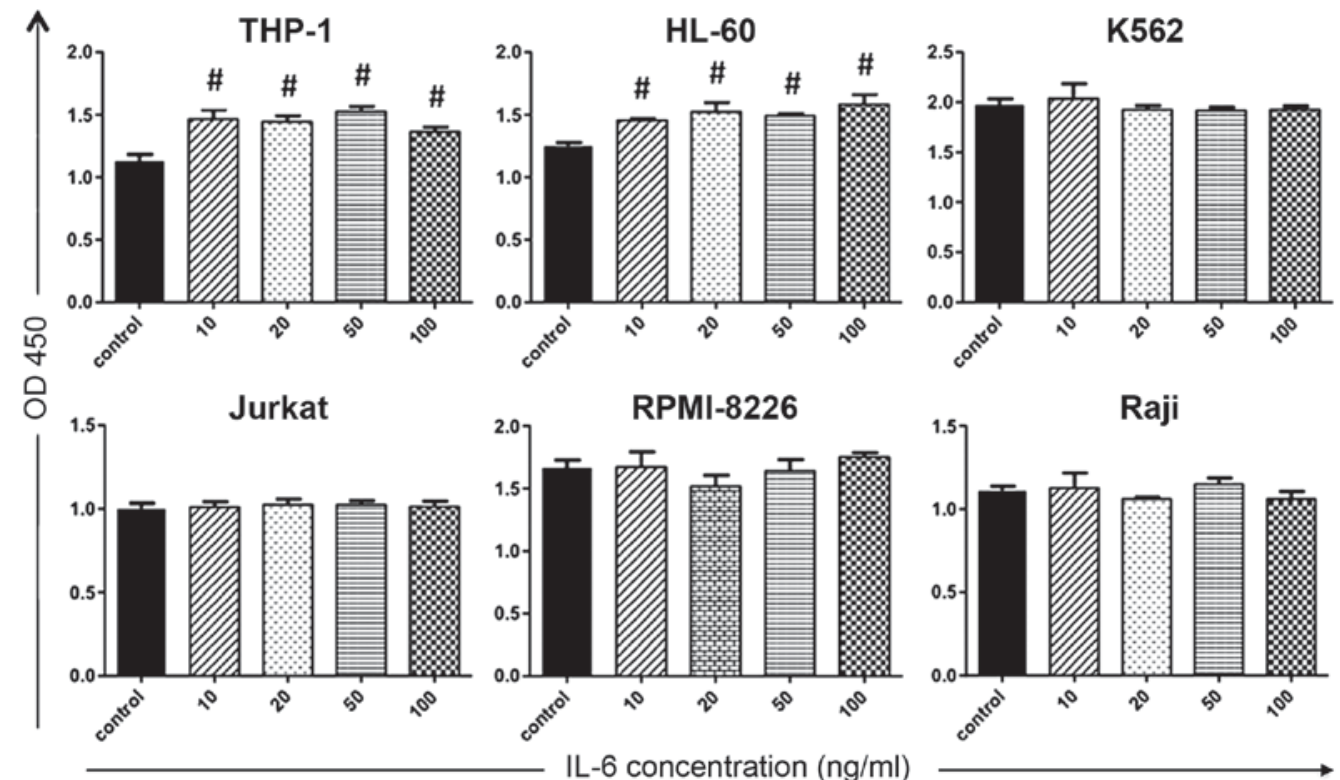

Figure 5. Exogenous IL-6 promotes the proliferation of THP-1 and HL-60 cells. Tumor cells were cultured in medium supplemented with 0, 10, 20, 50 or $100 \mathrm{ng} / \mathrm{ml}$ of recombinant human IL- 6 for $72 \mathrm{~h}$; cell proliferation was assessed by a Cell Counting kit- 8 assay. The experiments were repeated three times. The data are expressed as the means \pm standard deviation. ${ }^{*} \mathrm{P}<0.05$, increase vs. control. IL-6, interleukin- 6 . 
has exhibited clinical efficacy $(27,28)$. However, in the present study, the MM cell line RPMI-8226 exhibited no response to exogenous IL-6 despite expressing complete IL-6 receptors; proliferation was even inhibited by hUC-MSCs, which may be explained by a lack of signals downstream of IL-6, and by the expression of certain inhibitory cytokine receptors, as may also occur on leukemia cells.

Research on the effect of MSCs on lymphoma is controversial, possibly due to the different sources of MSCs and tumor cell types. Ahn et al (29) demonstrated that human adipose tissue derived-MSCs inhibited the growth of EL4 T-cell lymphoma cells by affecting the cell cycle and apoptosis. In contrast, in a study on mantle cell lymphoma, Medina et al (30) proposed that BM-MSCs may promote growth and migration, and inhibit apoptosis through the activation of the nuclear factor- $\kappa \mathrm{B}$ pathway. In the present study, hUC-MSCs promoted the growth of Raji Burkitt's lymphoma cells. Consistent with the study by Medina et al (30), growth promotion from cell-to-cell contact is likely to exhibit a notable effect on Raji cell growth, possibly due to a lack of cytokine receptor expression.

hUC-MSCs may also affect the secretion of tumor cells. A previous clinical study demonstrated that IL-6 and -10 levels were positively correlated in patients with lymphoma (31); exogenous IL-6 also increased the secretion of IL-10 by MM cells, and IL-10 promotes the development of MM in conjunction with IL-6 (32). The present study also revealed that hUC-MSCs promoted the secretion of IL-10 by MM and lymphoma cell lines, which may contribute to the secretion of IL-6.

Collectively, the results indicate a varying effect of hUC-MSCs on cells from various types of hematologic malignancy associated with cytokines and cell-to-cell contact depending on the expression of cytokine receptors on the cells. In particular, researchers applying hUC-MSCs in lymphoma should be aware of a potential tumor growth-promoting effect.

\section{Acknowledgements}

Not applicable.

\section{Funding}

The present study was supported by the NationalHigh-tech R\&D Program of China (863 Program; grant no. 2011AA020114).

\section{Availability of data and materials}

All data generated or analyzed during this study are included in this published article.

\section{Authors' contributions}

QL and YP performed the cytology experiments, and QL was a major contributor in writing the manuscript. TL and JX performed the cytometric bead array and flow cytometry experiments. YT performed the reverse transcription polymerase chain reaction experiments. $\mathrm{HC}$ and $\mathrm{BZ}$ conceived and designed the experiments, and gave final approval of the version to be published.All authors read and approved the final manuscript.

\section{Ethics approval and consent to participate}

The present study was approved by the Institutional Review Board of the Affiliated Hospital of the Academy of Military Medical Sciences (Beijing, China; protocol no., 2010-05-60). Written informed consent was obtained from all participants.

\section{Consent for publication}

Written informed consent for publication was obtained from all participants.

\section{Competing interests}

The authors declare that they have no competing interests.

\section{References}

1. Pittenger MF, Mackay AM, Beck SC, Jaiswal RK, Douglas R, Mosca JD, Moorman MA, Simonetti DW, Craig S and Marshak DR: Multilineage potential of adult human mesenchymal stem cells. Science 284: 143-147, 1999.

2. El Omar R, Beroud J, Stoltz JF, Menu P, Velot E and Decot V: Umbilical cord mesenchymal stem cells: The new gold standard for mesenchymal stem cell-based therapies? Tissue Eng Part B Rev 20: 523-544, 2014.

3. Nuschke A: Activity of mesenchymal stem cells in therapies for chronic skin wound healing. Organogenesis 10: 29-37, 2014.

4. Ra JC, Kang SK, Shin IS, Park HG, Joo SA, Kim JG, Kang BC, Lee YS, Nakama K, Piao M, et al: Stem cell treatment for patients with autoimmune disease by systemic infusion of culture-expanded autologous adipose tissue derived mesenchymal stem cells. J Transl Med 9: 181, 2011.

5. Bhansali A, Asokumar P, Walia R, Bhansali S, Gupta V, Jain A, Sachdeva N, Sharma RR, Marwaha N and Khandelwal N: Efficacy and safety of autologous bone marrow-derived stem cell transplantation in patients with type 2 diabetes mellitus: A randomized placebo-controlled study. Cell Transplant 23: 1075-1085, 2014.

6. Knaän-Shanzer S: Concise review: The immune status of mesenchymal stem cells and its relevance for therapeutic application. Stem Cells 32: 603-608, 2014.

7. Castro-Manrreza ME and Montesinos JJ: Immunoregulation by mesenchymal stem cells: Biological aspects and clinical applications. J Immunol Res 2015: 394917, 2015.

8. Vaes B, Van't Hof W, Deans R and Pinxteren J: Application of MultiStem(®) allogeneic cells for immunomodulatory therapy: Clinical progress and Pre-clinical challenges in prophylaxis for graft versus host disease. Front Immunol 3: 345, 2012.

9. Zhang L, Xiang $\mathrm{J}$ and Li G: The uncertain role of unmodified mesenchymal stem cells in tumor progression: What master switch? Stem Cell Res Ther 4: 22, 2013.

10. Hong IS, Lee HY and Kang KS: Mesenchymal stem cells and cancer: Friends or enemies? Mutat Res 768: 98-106, 2014.

11. Yang X, Hou J, Han Z, Wang Y, Hao C, Wei L and Shi Y: One cell, multiple roles: Contribution of mesenchymal stem cells to tumor development in tumor microenvironment. Cell Biosci 3: 5, 2013.

12. Ning H, Yang F, Jiang M, Hu L, Feng K, Zhang J, Yu Z, Li B, Xu C, Li Y, et al: The correlation between cotransplantation of mesenchymal stem cells and higher recurrence rate in hematologic malignancy patients: Outcome of a pilot clinical study. Leukemia 22: 593-599, 2008.

13. Nomoto-Kojima N, Aoki S, Uchihashi K, Matsunobu A, Koike E, Ootani A, Yonemitsu N, Fujimoto K and Toda S: Interaction between adipose tissue stromal cells and gastric cancer cells in vitro. Cell Tissue Res 344: 287-298, 2011.

14. Seo SH, Kim KS, Park SH, Suh YS, Kim SJ, Jeun SS and Sung YC: The effects of mesenchymal stem cells injected via different routes on modified IL-12-mediated antitumor activity. Gene Ther 18: 488-495, 2011.

15. Sarmadi VH, Tong CK, Vidyadaran S, Abdullah M, Seow HF and Ramasamy R: Mesenchymal stem cells inhibit proliferation of lymphoid origin haematopoietic tumour cells by inducing cell cycle arrest. Med J Malaysia 65: 209-214, 2010. 
16. Carrade DD, Lame MW, Kent MS, Clark KC, Walker NJ and Borjesson DL: Comparative analysis of the immunomodulatory properties of equine Adult-derived mesenchymal stem cells(). Cell Med 4: 1-11, 2012.

17. Ren G, Zhang L, Zhao X, Xu G, Zhang Y, Roberts AI, Zhao RC and Shi Y: Mesenchymal stem cell-mediated immunosuppression occurs via concerted action of chemokines and nitric oxide. Cell Stem Cell 2: 141-150, 2008.

18. Hu J, Yang Z, Wang J, Tang Y, Liu H, Zhang B and Chen H: Infusion of Trx-1-overexpressing hucMSC prolongs the survival of acutely irradiated NOD/SCID mice by decreasing excessive inflammatory injury. PLoS One 8: e78227, 2013.

19. Jones SA, Scheller J and Rose-John S: Therapeutic strategies for the clinical blockade of IL-6/gp130 signaling. J Clin Invest 121: 3375-3383, 2011.

20. Batsali AK, Kastrinaki MC, Papadaki HA and Pontikoglou C: Mesenchymal stem cells derived from Wharton's Jelly of the umbilical cord: Biological properties and emerging clinical applications. Curr Stem Cell Res Ther 8: 144-155, 2013.

21. Ramasamy R, Lam EW, Soeiro I, Tisato V, Bonnet D and Dazzi F: Mesenchymal stem cells inhibit proliferation and apoptosis of tumor cells: Impact on in vivo tumor growth. Leukemia 21: 304-310, 2007.

22. Fonseka M, Ramasamy R, Tan BC and Seow HF: Human umbilical cord blood-derived mesenchymal stem cells (hUCB-MSC) inhibit the proliferation of K562 (human erythromyeloblastoid leukaemic cell line). Cell Biol Int 36 : 793-801, 2012

23. Bozok Cetintas V, Aktug H, Oltulu F, Keskinoglu A, Erer Del Castello B and Taskiran D: The effects of mesenchymal stem cells on lymphoblastic leukemia cell proliferation. J BUON 19: 1006-1017, 2014

24. Zhu Y, Sun Z, Han Q, Liao L, Wang J, Bian C, Li J, Yan X, Liu Y, Shao $C$ and Zhao RC: Human mesenchymal stem cells inhibit cancer cell proliferation by secreting DKK-1. Leukemia 23: 925-933, 2009.

25. Rosean TR, Tompkins VS, Tricot G, Holman CJ, Olivier AK, Zhan F and Janz S: Preclinical validation of interleukin 6 as a therapeutic target in multiple myeloma. Immunol Res 59: $188-202,2014$
26. Klein B, Tarte K, Jourdan M, Mathouk K, Moreaux J, Jourdan E, Legouffe E, De Vos J and Rossi JF: Survival and proliferation factors of normal and malignant plasma cells. Int J Hematol 78: 106-113, 2003

27. Voorhees PM, Manges RF, Sonneveld P, Jagannath S, Somlo G, Krishnan A, Lentzsch S, Frank RC, Zweegman S, Wijermans PW, et al: A phase 2 multicentre study of siltuximab, an anti-interleukin-6 monoclonal antibody, in patients with relapsed or refractory multiple myeloma. Br J Haematol 161: 357-366, 2013.

28. Matsuyama Y, Nagashima T, Honne K, Kamata Y, Iwamoto M, Okazaki H, Sato K, Ozawa K and Minota S: Successful treatment of a patient with rheumatoid arthritis and Ig A-k multiple myeloma with tocilizumab. Intern Med 50: 639-642, 2011.

29. Ahn JO, Chae JS, Coh YR, Jung WS, Lee HW, Shin IS, Kang SK and Youn HY: Human adipose tissue-derived mesenchymal stem cells inhibit T-cell lymphoma growth in vitro and in vivo. Anticancer Res 34: 4839-4847, 2014.

30. Medina DJ, Goodell L, Glod J, Gelinas C, Rabson AB and Strair RK: Mesenchymal stromal cells protect mantle cell lymphoma cells from spontaneous and drug-induced apoptosis through secretion of B-cell activating factor and activation of the canonical and non-canonical nuclear factor $\mathrm{kB}$ pathways. Haematologica 97: 1255-1263, 2012.

31. Guney N, Soydinc HO, Basaran M, Bavbek S, Derin D, Camlica H, Yasasever V and Topuz E: Serum levels of interleukin-6 and interleukin-10 in Turkish patients with aggressive non-Hodgkin's lymphoma. Asian Pac J Cancer Prev 10: 669-674, 2009.

32. Kovacs E: Interleukin-6 leads to interleukin-10 production in several human multiple myeloma cell lines. Does interleukin-10 enhance the proliferation of these cells? Leuk Res 34: 912-916, 2010.

c) () $\Theta$ This work is licensed under a Creative Commons Attribution-NonCommercial-NoDerivatives 4.0 International (CC BY-NC-ND 4.0) License. 